CERN-TH/96-275

hep-th/9610018

\title{
Supermembrane dynamics from multiple interacting strings
}

\author{
J.G. Russo * \\ Theory Division, CERN \\ CH-1211 Geneva 23, Switzerland
}

\begin{abstract}
The supermembrane theory on $\mathbf{R}^{10} \times S^{1}$ is investigated, for membranes that wrap once around the compact dimension. The Hamiltonian can be organized as describing $N_{s}$ interacting strings, the exact supermembrane corresponding to $N_{s} \rightarrow \infty$. The zero-mode part of $N_{s}-1$ strings turn out to be precisely the modes which are responsible of instabilities. For sufficiently large compactification radius $R_{0}$, interactions are negligible and the lowest-energy excitations are described by a set of harmonic oscillators. We compute the physical spectrum to leading order, which becomes exact in the limit $g^{2} \rightarrow \infty$, where $g^{2} \equiv 4 \pi^{2} T_{3} R_{0}^{3}$ and $T_{3}$ is the membrane tension. As the radius is decreased, more strings become strongly interacting and their oscillation modes get frozen. In the zero-radius limit, the spectrum is constituted of the type IIA superstring spectrum, plus an infinite number of extra states associated with flat directions of the quartic potential.
\end{abstract}

October 1996

* e-mail: jrusso@vxcern.cern.ch 


\section{Introduction}

It is expected that supermembrane theories [1] will play a central role in a new formulation of string theory that should incorporate features of the strong coupling dynamics, which is now referred to as M-theory [2]. In the eleven-dimensional supermembrane theory, the existence of a supersymmetric ground state, together with the assumption of an energy gap to the first excited state, would be sufficient to guarantee that the low-energy limit of the theory will indeed be given by 11D supergravity, believed to govern the strong coupling limit of type IIA superstring theory [3,4. Moreover, supermembrane theory is known to be related to type IIA superstring theory by means of a procedure called double-dimensional reduction [5]. The idea is to consider the target space $\mathbf{R}^{10} \times S^{1}$, fix a gauge where the compact coordinate is proportional to one of the world-volume coordinates, say $\rho$, and -in the spirit of Kaluza-Klein reduction- assume that for small radius $R_{0}$ the relevant modes of the non-compact coordinates will be those independent of $\rho$. In this work we will incorporate the dynamics of other modes in a systematic way.

Strictly speaking, the statement that type IIA superstring theory is obtained from the supermembrane at small radius still remains to be proved. The actual statement of ref. [5] is that type IIA superstring theory is obtained under the ad hoc assumption that the non-compact coordinates are $\rho$-independent. As we will see, the problem is that there are extra states, associated with the instabilities of the quantum supermembrane on $\mathbf{R}^{11}$, which do not decouple as $R_{0} \rightarrow 0$. This will be clear in the present formulation, where the supermembrane will be organized as a system of interacting strings. In this language,

the extra states include the states associated with instabilities on $\mathbf{R}^{10} \times S^{1}$, which are constructed by using the zero-mode part of $N_{s}-1$ strings. In order for standard supermembrane theory to have a discrete mass spectrum, these zero modes have to be removed by hand from the original Hamiltonian. Since the action of [1] is not renormalizable, it is conceivable that in a correct description, appropriate to $R_{0}$ less than the Planck length, the unwanted states could decouple. Here we will keep all the modes of supermembrane theory, except at the end of sect. 6 , where we will consider the truncated Hamiltonian with the zero modes of $N_{s}-1$ strings removed. It describes a stable system, with a calculable discrete spectrum at large radius.

Another possibility [6,7] is that quantum effects could modify the asymptotic zeropoint energy in certain directions, in such a way that all modes remain confined, and as 
a result the Hamiltonian spectrum would be discrete. The problem is how to do this maintaining supersymmetry. The mechanism explored in ref. [7] uses a target-space with a boundary, where the membrane wave function is required to vanish. This requirement indeed removes the instabilities, but, on the face of it, it seems to also preclude the existence of massless states in the spectrum. Unfortunately, the general ground-state wave-functional is unknown, so it is not clear whether there could remain some eigenstate of zero energy satisfying the boundary condition.

In standard Kaluza-Klein theory, the extra dimension emerges in scattering processes involving energies greater than the inverse compactification radius, $1 / R_{0}$, i.e. when KaluzaKlein modes can be excited. Processes involving energies which are much smaller than $1 / R_{0}$, can be effectively described in terms of a $D-1$ dimensional theory. In the present case, one would like to see whether for energies much lower than $1 / R_{0}$ the theory can be effectively described in terms of a string theory, i.e. whether the only states that can be excited at $E \ll 1 / R_{0}$ correspond to oscillation modes of a string. In what follows, when discussing the regime $R_{0}^{2} \ll \alpha^{\prime}$, by "low-energy" we mean $E^{2} \leq O\left(1 / \alpha^{\prime}\right) \ll 1 / R_{0}^{2}$. This is the regime where we would expect to have a string theory description, with all the extra quantum states of the supermembrane frozen.

The dynamics dictated by the membrane Hamiltonian will be investigated in sects. 4 and 5. Although the full Hamiltonian is highly non-linear, the qualitative behavior of the system can be followed for all radii and, remarkably, exact statements about the relevant excitations of the system can be done in the extreme limits $R_{0}=\infty$ and $R_{0}=0$; from the ten-dimensional viewpoint, these correspond respectively to infinite and zero string coupling $g^{2} \equiv 4 \pi^{2} T_{3} R_{0}^{3}$. In the $R_{0}=\infty$ limit, with $T \equiv 2 \pi R_{0} T_{3}$ fixed, the Hamiltonian describes a system of decoupled harmonic oscillators. For finite but large $R_{0}$, quantum states with $\alpha^{\prime} M^{2} \ll O\left(g^{2}\right)$ will describe harmonic motion, while those with $\alpha^{\prime} M^{2}>O\left(g^{2}\right)$ will be affected by the full non-linearities of the supermembrane theory. In the limit $R_{0} \rightarrow 0$, the quantum states with non-trivial oscillator content in the compact direction will have $M^{2} \sim 1 / R_{0}^{2}$, and they get frozen: it takes an infinite energy to excite them. 


\section{Supermembrane Hamiltonian and instabilities}

\subsection{Generalities}

We will consider membranes of toroidal topologies. A complete set of functions on the torus is given by

$$
Y_{\mathbf{n}}(\sigma, \rho)=e^{i n_{1} \sigma+i n_{2} \rho}, \quad \mathbf{n}=\left(n_{1}, n_{2}\right), \quad \sigma, \rho \in[0,2 \pi) .
$$

The Lie bracket of $Y_{\mathbf{n}}(\sigma)$ corresponds to the structure constants of the area-preserving diffeomorphisms of the torus,

$$
\left\{Y_{\mathbf{n}}, Y_{\mathbf{n}^{\prime}}\right\}=f_{\mathbf{n} \mathbf{n}^{\prime} \mathbf{m}} Y^{\mathbf{m}}=-\left(\mathbf{n} \times \mathbf{n}^{\prime}\right) Y_{\mathbf{n}+\mathbf{n}^{\prime}}
$$

where

$$
\{X, Y\}=\partial_{\sigma} X \partial_{\rho} Y-\partial_{\rho} X \partial_{\sigma} Y, \quad \mathbf{n} \times \mathbf{n}^{\prime}=n_{1} n_{2}^{\prime}-n_{2} n_{1}^{\prime} .
$$

There is a truncated version of this infinite dimensional algebra, which approximates eq. (2.2) in the limit $N \rightarrow \infty$ :

$$
\left[J_{\mathbf{n}}, J_{\mathbf{n}^{\prime}}\right]=g_{\mathbf{n} \mathbf{n}^{\prime} \mathbf{m}} J^{\mathbf{m}}=-\frac{N}{2 \pi} \sin \frac{2 \pi}{N}\left(\mathbf{n} \times \mathbf{n}^{\prime}\right) J_{\mathbf{n}+\mathbf{n}^{\prime}} .
$$

One can restrict to a fundamental lattice defined by $n_{1}, n_{2}=0, \ldots, N-1$, with the exception of the origin $n_{1}=n_{2}=0$, which label $N^{2}-1$ generators; these can be shown to span the algebra of $S U(N)$ [8]. Note that the Cartan subalgebra can be taken to be generated by $J_{\mathbf{n}}$, with $n_{1}=0, n_{2}=1, \ldots, N-1$.

Taking $X^{9}$ as one of the light-cone variables, $X^{ \pm}=\frac{X^{0} \pm X^{9}}{\sqrt{2}}$, the light-cone Hamiltonian of the supermembrane is given by 9,10

$$
\begin{gathered}
H=H_{\mathrm{B}}+H_{\mathrm{F}}, \\
H_{\mathrm{B}}=2 \pi^{2} \int d \sigma d \rho\left[P_{a}^{2}+\frac{T_{3}^{2}}{2}\left(\left\{X^{a}, X^{b}\right\}\right)^{2}\right], \\
H_{\mathrm{F}}=-T_{3} p_{0}^{+} \int d \sigma d \rho \bar{\theta} \Gamma^{a}\left\{X^{a}, \theta\right\}, \quad a=1,2, \ldots, 8,10 .
\end{gathered}
$$

Here $\theta$ are real $S O(9)$ spinors $\theta_{\alpha}, \alpha=1, \ldots, 16$. The mass square operator $M^{2}=2 p_{0}^{+} p_{0}^{-}-$ $\left(p_{0}^{a}\right)^{2}$ is given by $M^{2}=2 H-\left(p_{0}^{a}\right)^{2} . T_{3}$ is the membrane tension, $\left[T_{3}\right]=\mathrm{cm}^{-3}$.

The Hamiltonian has a residual gauge invariance, corresponding to the symmetry (2.2) of area-preserving diffeomorphisms of the torus. Let us choose $Y_{\left(0, n_{2}\right)}$ as the generators of the Cartan subalgebra $K$. We expand

$$
\begin{gathered}
X^{a}(\sigma, \rho)=\sum_{\mathbf{n}} X_{\mathbf{n}}^{a} Y_{\mathbf{n}}(\sigma, \rho)=X_{K}^{a}(\sigma, \rho)+\tilde{X}^{a}(\sigma, \rho), \\
X_{K}^{a}(\sigma, \rho)=\sum_{\mathbf{n} \in K} X_{\mathbf{n}}^{a} Y_{\mathbf{n}}(\sigma, \rho), \quad \tilde{X}^{a}(\sigma, \rho)=\sum_{\mathbf{n}, n_{1} \neq 0} X_{\mathbf{n}}^{a} Y_{\mathbf{n}}(\sigma, \rho) .
\end{gathered}
$$

The area-preserving gauge symmetry can be fixed by setting $\tilde{X}^{10}(\sigma, \rho)=0$ [6]. 


\subsection{Instabilities in the presence of compact dimensions}

Consider the target space $\mathbf{R}^{9} \times S^{1} \times S^{1}$, and expand the single-valued membrane coordinates

$$
X^{a}(\sigma, \rho)=\sum_{\mathbf{n}} X_{\mathbf{n}}^{a} Y_{\mathbf{n}}(\sigma, \rho), \quad a=1, \ldots, 8 .
$$

Let the coordinates $X^{9}, X^{10}$ have periods $2 \pi R_{9}$ and $2 \pi R_{10}$, respectively. Now we perform a semiclassical expansion around a classical configuration with non-trivial winding numbers,

$$
\begin{gathered}
X^{9}(\sigma, \rho)=w_{1} R_{9} \sigma+w_{2} R_{9} \rho+\sum_{\mathbf{n}} X_{\mathbf{n}}^{9} Y_{\mathbf{n}}(\sigma, \rho) \\
X^{10}(\sigma, \rho)=v_{1} R_{10} \sigma+v_{2} R_{10} \rho+\sum_{\mathbf{n}} X_{\mathbf{n}}^{10} Y_{\mathbf{n}}(\sigma, \rho) .
\end{gathered}
$$

The small-oscillation spectrum in the case $w_{2}=v_{1}=0$ was derived in ref. [11]. As in sect. 2.1, the light-cone gauge can be used to remove the single-valued part of $X^{9}$, and the residual symmetry of area-preserving diffeomorphisms can be fixed as before by constraining $X^{10}$ to live in the Cartan subspace of the algebra (2.2), that is

$$
\begin{gathered}
X^{9}(\sigma, \rho)=w_{1} R_{9} \sigma+w_{2} R_{9} \rho+X_{(0,0)}^{9} \\
X^{10}(\sigma, \rho)=v_{1} R_{10} \sigma+v_{2} R_{10} \rho+X_{K}^{10}(\sigma, \rho), \quad X_{K}^{10}(\sigma, \rho)=\sum_{\mathbf{n} \in K} X_{\mathbf{n}}^{10} Y_{\mathbf{n}}(\sigma, \rho)
\end{gathered}
$$

The winding number that counts how many times the toroidal membrane is wrapped around the target-space torus is given by

$$
w_{0}=\frac{1}{4 \pi^{2} R_{9} R_{10}} \int d \sigma d \rho\left\{X^{9}, X^{10}\right\}=w_{1} v_{2}-w_{2} v_{1}
$$

For $w_{0} \neq 0$ the membrane is expected to be stable for topological reasons. Let us see how this works using the Hamiltonian formulation. Because of the winding contributions, there will now be new terms in the Hamiltonian, which will in turn be responsible for the confinement of all membrane modes. In particular, the bosonic interaction term is now given by

$$
\begin{gathered}
\pi^{2} T_{3}^{2} \int d \sigma d \rho\left(\left\{X^{\mu}, X^{\nu}\right\}\right)^{2}=\pi^{2} T_{3}^{2} \int d \sigma d \rho\left(\left\{X^{a}, X^{b}\right\}\right)^{2} \\
+4 \pi^{2} T_{3}^{2} R_{10} \int d \sigma d \rho\left\{X^{10}, X^{i}\right\}\left(v_{2} \partial_{\sigma} X^{i}-v_{1} \partial_{\rho} X^{i}\right)
\end{gathered}
$$




$$
+8 \pi^{4} T_{3}^{2}\left(\sum_{\mathbf{n}}\left[R_{9}^{2}(\mathbf{w} \times \mathbf{n})^{2} X_{\mathbf{n}}^{a} X_{-\mathbf{n}}^{a}+R_{10}^{2}(\mathbf{v} \times \mathbf{n})^{2} X_{\mathbf{n}}^{a} X_{-\mathbf{n}}^{a}\right]+R_{9}^{2} R_{10}^{2}(\mathbf{w} \times \mathbf{v})^{2}\right) .
$$

Here $\mu, \nu=1, \ldots, 10$ and $a, b=1, \ldots, 8,10$, where the replacement $X^{10} \rightarrow X_{K}^{10}$ is understood on the right-hand side. Let us choose $Y_{\mathbf{v}}$ as one of the Cartan generators. Instabilities due to flat directions in the full Hamiltonian can only occur along the Cartan directions [6]; for all $\mathbf{n} \in K$ we will have $\mathbf{v} \times \mathbf{n}=0$. Let us take for example $v_{1}=0, v_{2}=1$, so that the Cartan subalgebra will be generated by $Y_{\left(0, n_{2}\right)}$. Provided we choose w outside the Cartan subspace, that is $\mathbf{w} \times \mathbf{v}=w_{1} \neq 0$, then the term in eq. (2.13) containing $(\mathbf{w} \times \mathbf{n})^{2}$ will be non-zero for all Cartan directions $\mathbf{n}=\left(0, n_{2}\right)$ and, as a result, all modes will be confined. Thus, as expected, the Hamiltonian will have a discrete spectrum precisely when the membrane is topologically protected, i.e. $w_{0}=\mathbf{w} \times \mathbf{v} \neq 0$. Note that this is true in the large radius regime where the semiclassical approximation is justified (for small radius, world-volume instanton effects may lead to instabilities).

If the target space is given by $\mathbf{R}^{10} \times S^{1}$, then the stability of the membrane cannot be topologically protected. It is clear from the above analysis that there will be flat directions: there is only one term $\frac{1}{2} R_{10}^{2}(\mathbf{v} \times \mathbf{n})^{2} X_{\mathbf{n}}^{a} X_{-\mathbf{n}}^{a}$, which vanishes for certain $\mathbf{n}$. In particular, taking $\mathbf{v}=(0,1)$, wave packets of the form $\chi\left(X_{\left(0, n_{2}\right)}-V t\right)$, with $\chi$ of compact support, can escape to infinity.

\section{Hamiltonian for multiple strings}

\subsection{Strings from membranes}

Let the target space be given by $\mathbf{R}^{10} \times S^{1}$, with $X^{10}$ representing the compact dimension, and take $X^{9}$ as one of the light-cone variables,

$$
X^{ \pm}=\frac{X^{0} \pm X^{9}}{\sqrt{2}}
$$

Consider a configuration that wraps once around $S^{1}$, and fix the symmetry of areapreserving diffeomorphisms by setting

$$
X^{10}=R_{0} \rho+X_{K}^{10}(\rho)
$$


where $X_{K}^{10}(\rho)$ is a single-valued function that lives in the Cartan subspace generated by $Y_{(0, m)}$,

$$
X_{K}^{10}(\rho)=\sum_{m} X_{(0, m)}^{10} e^{i m \rho}
$$

Let us write $P^{10}(\sigma, \rho)=P_{K}^{10}+\tilde{P}^{10}$, where $P_{K}^{10}$ lives in the Cartan subspace, and $\tilde{P}^{10}$ in the complement. As usual, the constraints associated with the gauge symmetry can be either imposed on the quantum states or they can be solved at the classical level to eliminate one of the variables as an independent degree of freedom to be quantized. A description of the local and global constraints in the light-cone gauge can be found in the appendix. The local constraints can be solved for $\tilde{P}^{10}$ in terms of $X_{K}^{10}$ and the transverse coordinates and momenta $X^{i}, P^{i}, i=1, \ldots, 8$ (see eqs. (A.6), (A.8)).

In the following, we will concentrate on the bosonic part. The incorporation of fermions is straightforward and will be discussed in sect. 6. Using eq. (3.1), the Hamiltonian takes the form $\left(f^{\prime}(\sigma) \equiv \partial_{\sigma} f(\sigma)\right.$ throughout)

$$
\begin{gathered}
H_{\mathrm{B}}=H_{0}+H_{1}, \\
H_{0}=2 \pi^{2} \int d \sigma d \rho\left[P_{a}^{2}+T_{3}^{2} R_{0}^{2}\left(X_{i}^{\prime}\right)^{2}\right], \\
H_{1}=\pi^{2} T_{3}^{2} \int d \sigma d \rho\left[\left(\left\{X^{a}, X^{b}\right\}\right)^{2}+4 R_{0} \partial_{\rho} X^{10}\left(X_{i}^{\prime}\right)^{2}\right], \\
a, b=1, \ldots, 8,10, \quad i, j=1, \ldots, 8,
\end{gathered}
$$

understanding $X^{10} \rightarrow X_{K}^{10}(\rho), P^{10} \rightarrow P_{K}^{10}(\rho)+\tilde{P}^{10}$, where $\tilde{P}^{10}$ is given by eq. (A.6).

Let us now expand the transverse bosons in a complete basis of functions on $S^{1}$ :

$$
\begin{gathered}
X^{i}(\sigma, \rho, \tau)=\sum_{m} X_{m}^{i}(\sigma, \tau) e^{i m \rho}, \quad P^{i}(\sigma, \rho, \tau)=\frac{1}{2 \pi} \sum_{m} P_{m}^{i}(\sigma, \tau) e^{i m \rho} \\
{\left[X_{m}^{i}(\sigma), P_{n}^{j}\left(\sigma^{\prime}\right)\right]=i \delta_{m+n} \delta^{i j} \delta\left(\sigma-\sigma^{\prime}\right), \quad X_{m}^{\dagger}=X_{-m}, \quad P_{m}^{\dagger}=P_{-m} .}
\end{gathered}
$$

Hermitian variables $Y_{m}^{(1,2)}$ can be introduced through $X_{ \pm m}=Y_{m}^{(1)} \pm i Y_{m}^{(2)}, m=1,2, \ldots$, and similarly for $P_{m}$.

Inserting the expansions (3.5), the Hamiltonian (3.2) reduces to the following expressions:

$$
H_{0}=\pi T^{2} \int d \sigma \sum_{m}\left[T^{-2} P_{m}^{a} P_{-m}^{a}+X_{m}^{a \prime} X_{-m}^{a \prime}\right], \quad T \equiv 2 \pi R_{0} T_{3}
$$




$$
\begin{aligned}
H_{1}= & \frac{\pi T^{2}}{R_{0}^{2}} \int d \sigma \sum_{m, n, p}\left[m p\left(X_{n}^{a \prime} X_{p}^{a}\right)\left(X_{-m-n-p}^{b \prime} X_{m}^{b}\right)-\right. \\
& \left.n p\left(X_{n}^{a} X_{p}^{a}\right)\left(X_{-m-n-p}^{b \prime} X_{m}^{b \prime}\right)\right] \\
+ & \frac{2 \pi i T^{2}}{R_{0}} \int d \sigma \sum_{m, n} m X_{(0, m)}^{10} X_{n}^{i \prime} X_{-n-m}^{i \prime}
\end{aligned}
$$

In this context, the usual double-dimensional reduction procedure [5] corresponds to dropping all modes $X_{m}^{a}(\sigma)$ with $m \neq 0$, and setting the Kaluza-Klein momentum $p^{10}=\int d \rho P^{10}(\rho)$ to zero, so what remains is

$$
\alpha^{\prime} H_{\mathrm{B}}^{\mathrm{red}}=\frac{T}{2} \int d \sigma\left[T^{-2} P_{0}^{i} P_{0}^{i}+X_{0}^{i^{\prime}} X_{0}^{i^{\prime}}\right], \quad \alpha^{\prime} \equiv(2 \pi T)^{-1} .
$$

Upon the identification of $T$ with the standard string tension, this is exactly the string theory Hamiltonian.

World-volume time translations are generated by $\tilde{H}=\alpha^{\prime}\left(H_{0}+H_{1}\right)$. From the Hamilton equations, one obtains

$$
P_{m}^{i}=T \partial_{\tau} X_{m}^{i}
$$

Regarding $H_{1}$ as a perturbation, the equations of motion of the unperturbed Hamiltonian $H_{0}$ give

$$
\partial_{\sigma}^{2} X_{m}^{i}=\partial_{\tau}^{2} X_{m}^{i}
$$

The solution satisfying the periodicity condition $X^{a}(\sigma+2 \pi)=X^{a}(\sigma)$ is given by

$$
\begin{gathered}
X_{m}^{i}(\sigma, \tau)=x_{m}^{i}+\alpha^{\prime} p_{m}^{i} \tau+i \sqrt{\frac{\alpha^{\prime}}{2}} \sum_{k \neq 0} \frac{1}{k}\left(\alpha_{(k, m)}^{i} e^{-i k(\tau-\sigma)}+\tilde{\alpha}_{(k, m)}^{i} e^{-i k(\tau+\sigma)}\right), \\
{\left[\alpha_{(k, m)}^{i}, \alpha_{(l, n)}^{j}\right]=k \delta_{k+l} \delta_{m+n} \delta^{i j}} \\
\alpha_{(k, m)}^{\dagger}=\alpha_{(-k,-m)}, \quad x_{m}^{\dagger}=x_{-m}, \quad p_{m}^{\dagger}=p_{-m} .
\end{gathered}
$$

Throughout, indices $m, n$ are used for Fourier modes in $\rho$, whereas $k, l$ are associated with Fourier modes in $\sigma$.

The wave function of the unperturbed Hamiltonian $H_{0}$ is just the product of an infinite set of harmonic oscillator wave functions. The picture of multiple weakly interacting strings is a convenient description only in the large radius regime. In sect. 5 we will examine under which conditions can $H_{1}$ be treated as a perturbation, and what are the relevant degrees of freedom as the radius is gradually changed. 


\subsection{Instability modes}

Taking $Y_{(0,1)}$ as a Cartan generator, it follows from the discussion of sect. 2 that our system will have potential valleys along $X_{(0, n)}^{a}$, corresponding to the constant modes in the coordinate $\sigma$. This holds for any value of the radius $R_{0}$ (see also sects. 4 and 5 ). Let us see what this implies for the solution (3.10), valid for large $R_{0}$. Inserting (3.10) into eq. (3.5), we find

$$
\begin{gathered}
X^{a}(\sigma, \rho, \tau)=x_{0}^{a}+\alpha^{\prime} p_{0}^{a} \tau+x^{a}(\rho)+2 \pi \alpha^{\prime} p^{a}(\rho) \tau \\
+i \sqrt{\frac{\alpha^{\prime}}{2}} \sum_{k \neq 0} \frac{1}{k}\left(\alpha_{k}^{a}(\rho) e^{-i k(\tau-\sigma)}+\tilde{\alpha}_{k}^{a}(\rho) e^{-i k(\tau+\sigma)}\right),
\end{gathered}
$$

where

$$
\begin{gathered}
x^{a}(\rho)=\sum_{m \neq 0} x_{m}^{a} e^{i m \rho}, \quad p^{a}(\rho)=\frac{1}{2 \pi} \sum_{m \neq 0} p_{m}^{a} e^{i m \rho}, \\
\alpha_{k}^{i}(\rho)=\sum_{m} \alpha_{(k, m)}^{i} e^{i m \rho}, \quad \tilde{\alpha}_{k}^{i}(\rho)=\sum_{m} \tilde{\alpha}_{(k, m)}^{i} e^{i m \rho}, \quad \alpha_{k}^{10}=\tilde{\alpha}_{k}^{10}=0 .
\end{gathered}
$$

We have separated $x_{0}^{a}$ and $p_{0}^{a}$, representing the center-of-mass coordinate and momentum of the membrane,

$$
x_{0}^{a}+\alpha^{\prime} p_{0}^{a} \tau=\frac{1}{4 \pi^{2}} \int d \sigma d \rho X^{a}(\sigma, \rho), \quad p_{0}^{a}=\int d \sigma d \rho P^{a}(\sigma, \rho)
$$

Since the Cartan subspace is generated by $Y_{(0, m)}=e^{i m \rho}$, the modes that will cause the instabilities of the supermembrane are $x^{a}(\rho), p^{a}(\rho)$, i.e. there are flat directions along all $x_{m}^{a}$ with $m \neq 0$. [By virtue of translational invariance, the zero-mode of $X_{0}^{a}(\sigma, \tau)$ does not appear in the Hamiltonian; the center-of-mass momentum $\int d \sigma P_{0}^{a}$ is exactly conserved, and $\left(p_{0}^{a}\right)^{2}$ will later be absorbed into the Lorentz-invariant mass-squared operator.] It is important that the $x_{m}^{a}$ do not appear in $H_{0}$. These are indeed the only directions that are not stabilized by the winding contributions. It may be less obvious that the potential valleys remain in spite of their presence in $H_{1}$, but this can be understood by closer inspection of the potential (3.7) (see sect. 5). 


\section{A simple model}

There are two questions that we would now like to address. The first one concerns the regime of applicability of perturbation theory. The second, in a sense, complementary question is what modes can be safely dropped for a given radius $R_{0}$. Obviously, the modes $x_{m}^{a}$ cannot be dropped at any radius, since they can be excited by an arbitrarily small energy $\epsilon$. The essential features can be illustrated by the following Hamiltonian:

$$
\begin{gathered}
\mathcal{H}=\mathcal{H}_{0}+\mathcal{H}_{1}, \\
\mathcal{H}_{0}=\frac{1}{2 T^{2}}\left(p_{x_{1}}^{2}+p_{x_{2}}^{2}+p_{y}^{2}\right)+\frac{1}{2} x_{1}^{2}+\frac{1}{2} x_{2}^{2}, \\
\mathcal{H}_{1}=\frac{\lambda}{R_{0}^{2}}\left(x_{1}^{2} y^{2}+x_{2}^{2} y^{2}+x_{1}^{2} x_{2}^{2}\right), \quad \lambda>0 .
\end{gathered}
$$

The coordinate $y$ is the analogue of the $x_{m}^{a}$, corresponding to the Cartan generators $Y_{(0, m)}=e^{i m \rho}$. Classically, a mode can leak out to infinity along the direction $y$, with $x_{1}=x_{2}=0$. In the quantum theory, the purely bosonic system is stabilized thanks to a zero-point energy contribution from transverse fluctuations in the $x_{1}, x_{2}$ directions. In the supersymmetric system, this zero-point energy cancels out; as a result, a wave packet can move off to $y=\infty$, and the spectrum is continuous. Since these excitations can be produced by an infinitesimal energy, the $y$ direction does not get frozen at any radius $R_{0}$. The Hamiltonian (4.1) (more precisely, its supersymmetric version) will thus have a continuum spectrum of eigenvalues.

Now consider the truncated version $\mathcal{H}=\mathcal{H}_{0}+\mathcal{H}_{1}$, with $(T=1)$

$$
\mathcal{H}_{0}=\frac{1}{2}\left(p_{x_{1}}^{2}+p_{x_{2}}^{2}+x_{1}^{2}+x_{2}^{2}\right), \quad \mathcal{H}_{1}=\frac{\lambda}{R_{0}^{2}} x_{1}^{2} x_{2}^{2} .
$$

In terms of standard creation and annihilation operators,

$$
\begin{gathered}
\mathcal{H}_{0}=\frac{1}{2}\left(a_{1}^{\dagger} a_{1}+a_{1} a_{1}^{\dagger}+a_{2}^{\dagger} a_{2}+a_{2} a_{2}^{\dagger}\right), \\
\mathcal{H}_{1}=\frac{\lambda}{4 R_{0}^{2}}\left(a_{1}+a_{1}^{\dagger}\right)^{2}\left(a_{2}+a_{2}^{\dagger}\right)^{2} .
\end{gathered}
$$

Let us treat $\mathcal{H}_{1}$ as a perturbation, and write the eigenvalues of $\mathcal{H}$ as $E=E^{(0)}+E^{(1)}+\ldots$, with $E^{(0)}=n_{1}+n_{2}+1$. Since $\mathcal{H}_{0}$ has degenerate eigenvalues, to find $E^{(1)}$ we need to solve 
the secular equation. Denote by $m_{1}, m_{2}$ the positive integers satisfying $m_{1}+m_{2}+1=$ $E^{(0)}=$ fixed. Then the $E^{(1)}$ are obtained from the equation

$$
\left|\left\langle m_{1}, m_{2}\left|\mathcal{H}_{1}\right| m_{1}^{\prime}, m_{2}^{\prime}\right\rangle-E^{(1)} \delta_{m_{1} m_{1}^{\prime}} \delta_{m_{2} m_{2}^{\prime}}\right|=0
$$

where

$$
\begin{aligned}
\left\langle m_{1}, m_{2}\left|\mathcal{H}_{1}\right| m_{1}^{\prime}, m_{2}^{\prime}\right\rangle= & \frac{\lambda}{4 R_{0}^{2}}\left[\left(2 m_{1}+1\right) \delta_{m_{1} m_{1}^{\prime}}+\sqrt{\left(m_{1}+1\right)\left(m_{1}+2\right)} \delta_{m_{1}^{\prime}, m_{1}+2}+\right. \\
& \left.\sqrt{m_{1}\left(m_{1}-1\right)} \delta_{m_{1}^{\prime}, m_{1}-2}\right][1 \leftrightarrow 2] .
\end{aligned}
$$

Perturbation theory is applicable provided $E^{(0)} \gg E^{(1)}$ (more precisely, $\left.\psi^{(0)} \gg \psi^{(1)}\right)$. For low $E^{(0)}$, all $m_{1}$ and $m_{2}$ are small, and the solutions to (4.7) will be $E^{(1)}=O\left(\lambda / R_{0}^{2}\right)$. Thus for those states perturbation theory can be applied provided $\lambda / R_{0}^{2} \ll 1$. For high $E^{(0)}$, there will be matrix elements in (4.8) where both $m_{1}, m_{2} \gg 1$, so that the eigenvalues $E^{(1)}$ can be as high as $O\left(m_{1} m_{2} \lambda / R_{0}^{2}\right)$, with a maximum for $m_{1} \cong m_{2}$. Since $E^{(0)} \cong 2 m_{1}$, the use of perturbation theory is therefore justified only for $m_{1} \ll R_{0}^{2} / \lambda$. The oscillation amplitude for states with $m_{1}, m_{2} \gg R_{0}^{2} / \lambda$ is large and their motion is controlled by $\mathcal{H}_{1}$.

As the radius is decreased, the number of modes subject to harmonic motion will diminish. For $\lambda / R_{0}^{2}>1$, even the zero-point mode $n_{1}=n_{2}=0$ will be influenced by $\mathcal{H}_{1}$. The general behavior of the system can be understood by drawing equipotential lines and varying $\lambda / R_{0}^{2}$. For a given energy $E$, the classically accessible region is delimited by the curve

$$
\frac{1}{2}\left(x_{1}^{2}+x_{2}^{2}\right)+\frac{\lambda}{R_{0}^{2}} x_{1}^{2} x_{2}^{2}=E .
$$

At $E \ll R_{0}^{2} / \lambda$, this line is essentially a circle; the motion is harmonic, with an oscillation amplitude given by $\left\langle x_{1}^{2}\right\rangle,\left\langle x_{2}^{2}\right\rangle \cong E$. As $\lambda / R_{0}^{2}$ is increased, the circles are deformed, with a maximum decrease of the diameters on the axes $x_{1}= \pm x_{2}$. For $\lambda / R_{0}^{2} \rightarrow \infty$, the figure is extremely narrow, and the motion will be frozen, except along the axes $x_{1}=0, x_{2}=0$. It is easy to see that the only possible finite energy excitations will then be those in which the motion takes place only in one coordinate, $x_{1}$ or $x_{2}$, that is with $\left\langle x_{1}^{2} x_{2}^{2}\right\rangle \cong 0$. Indeed, a wave function $\psi$ where $\left\langle\psi\left|x_{1}^{2} x_{2}^{2}\right| \psi\right\rangle$ is non-vanishing will have an infinite energy, since

$$
E=\langle\psi|\mathcal{H}| \psi\rangle>\frac{\lambda}{R_{0}^{2}}\left\langle\psi\left|x_{1}^{2} x_{2}^{2}\right| \psi\right\rangle \rightarrow \infty \quad \text { as } \quad \frac{\lambda}{R_{0}^{2}} \rightarrow \infty .
$$

Thus at very small radius the system reduces to a one-dimensional harmonic oscillator. The only states relevant to low-energy $(E=O(1))$ physics have the form $|\psi\rangle=\delta\left(x_{1}\right)\left|\psi^{(0)}\left(x_{2}\right)\right\rangle$ or $|\psi\rangle=\delta\left(x_{2}\right)\left|\psi^{(0)}\left(x_{1}\right)\right\rangle$. 


\section{Membrane dynamics at different compactification radii}

The previous discussion can be generalized to our system, with $H_{0}$ and $H_{1}$ given by (3.6), (3.7). As discussed in sect. 3, the Hamiltonian has a continuum spectrum of eigenvalues due to the zero modes of the strings with $m \neq 0$. The constant parts of the $X_{m}^{a}(\sigma)$ play the same role as the coordinate $y$ of the model (4.1): they only appear in $H_{1}$, but their appearance is not sufficient to remove flat directions.

Let us expand $X_{m}^{a}(\sigma), P_{m}^{a}(\sigma)$ in terms of adimensional mode operators

$$
\begin{gathered}
X_{m}^{a}(\sigma)=\sqrt{\alpha^{\prime}} \sum_{k} X_{(k, m)}^{a} e^{i k \sigma}, \quad P_{m}^{a}(\sigma)=\frac{1}{2 \pi \sqrt{\alpha^{\prime}}} \sum_{k \neq 0} P_{(k, m)}^{a} e^{i k \sigma}, \\
X_{(k, m)}^{10}=P_{(k, m)}^{10}=0 \text { if } k \neq 0, \\
{\left[X_{(k, m)}^{a}, P_{\left(k^{\prime}, m^{\prime}\right)}^{b}\right]=i \delta_{m+m^{\prime}} \delta_{k+k^{\prime}} \delta^{a b},}
\end{gathered}
$$

where $\alpha^{\prime}$ has been introduced in eq. (3.8), and it is related to the membrane tension by $\alpha^{\prime}=\left(4 \pi^{2} R_{0} T_{3}\right)^{-1}$. In the free case, these mode operators will be given as linear combinations of $\alpha_{(k, m)}, \tilde{\alpha}_{(k, m)}$ operators, with simple exponential time dependence, as in eq. (3.10). By inserting the expansions (5.1) in eqs. (3.6) and (3.7) we obtain

$$
\begin{aligned}
\alpha^{\prime} H_{0}= & \frac{1}{2} \sum_{\mathbf{n}}\left[P_{\mathbf{n}}^{a} P_{-\mathbf{n}}^{a}+k^{2} X_{\mathbf{n}}^{i} X_{-\mathbf{n}}^{i}\right], \quad \mathbf{n} \equiv(k, m), \\
\alpha^{\prime} H_{1}= & \frac{1}{4 g^{2}} \sum_{\mathbf{n}_{1}, \mathbf{n}_{2}, \mathbf{n}_{3}}\left(\mathbf{n}_{1} \times \mathbf{n}_{2}\right)\left(\mathbf{n}_{3} \times \mathbf{n}_{4}\right) X_{\mathbf{n}_{1}}^{a} X_{\mathbf{n}_{2}}^{b} X_{\mathbf{n}_{3}}^{a} X_{\mathbf{n}_{4}}^{b} \\
& +\frac{i}{g} \sum_{k, m, n} m k^{2} X_{(0, m)}^{10} X_{(k, n)}^{i} X_{(-k,-m-n)}^{i}, \\
g^{2} \equiv & \frac{R_{0}^{2}}{\alpha^{\prime}}=4 \pi^{2} R_{0}^{3} T_{3}, \quad \mathbf{n}_{4}=-\mathbf{n}_{1}-\mathbf{n}_{2}-\mathbf{n}_{3} .
\end{aligned}
$$

In the infinite radius limit, $g^{2} \rightarrow \infty$, and the Hamiltonian becomes that of an infinite set of harmonic oscillators labelled by $(k, m)$. It is naturally organized as an infinite sum of free string theory Hamiltonians labelled by $m$, which is already manifiest in eq. (3.6). Strings with $m \neq 0$ are the analogue of Kaluza-Klein modes, which decouple from low-energy physics at small compactification radius.

Now the Hamiltonian is more complicated than that of the simplified model (4.1), since it contains an infinite number of terms and the structure (dictated by the group 
of area-preserving diffeomorphisms) is somewhat intricate. Nevertheless, it is possible to determine under what conditions a generic quantum state will be weakly coupled for a given (large) $g^{2}$. In terms of creation/annihilation operators, $H_{0}, H_{1}$ contain terms of the form

$$
\alpha^{\prime} H_{0}=k N_{(k, m)}+\ldots, \quad \alpha^{\prime} H_{1}=\frac{1}{4 g^{2}} \frac{(n l-m k)^{2}}{k l} N_{(k, n)} N_{(l, m)}+\ldots
$$

where $N_{(k, m)}=a_{(k, m)}^{\dagger} \cdot a_{(k, m)}$ is the occupation number of the oscillator $(k, m)$, and we are considering particular terms in $H_{1}$ with $l^{\prime}=-k$ and $p=-n$. Let us take

$$
n=m_{0} \bar{n}, \quad m=m_{0} \bar{m}, \quad N_{(k, n)}=N_{0} \bar{N}_{(k, n)}, \quad N_{(l, m)}=N_{0} \bar{N}_{(l, m)}
$$

where $\bar{n}, \bar{m}, \bar{N}_{(k, n)}, \bar{N}_{(l, m)}$ are $O(1)$ and $m_{0} \gg 1, N_{0} \gg 1$. Let us also consider $l \sim k \gg 1$ and $g^{2} \gg 1$. Such a quantum state is weakly coupled provided $H_{1} \ll H_{0}$, or

$$
\frac{1}{g^{2}} m_{0}^{2} N_{0} \ll k
$$

It is interesting to note that, for a given string $m_{0}$ and a given occupation number $N_{0}$, quantum states made of oscillators with higher frequency $k$ will describe a more harmonic motion. This is not a surprise, since the oscillation amplitudes go like $\left\langle X_{(k, m)}^{2}\right\rangle \sim N_{0} / k$, so the region of non-linearities is not reached for high enough $k$. States with low occupation number made of $a_{(k, m)}^{\dagger}$, with $m=O(1)$, will be governed by the harmonic oscillator Hamiltonian for all $k$ provided $g^{2} \gg 1$. From eq. (5.5) one also sees that for any given state $|\psi\rangle$, there exists a sufficiently large coupling $g^{2}$ so that $\left\langle\psi\left|H_{1}\right| \psi\right\rangle$ can be neglected as compared to $\left\langle\psi\left|H_{0}\right| \psi\right\rangle$.

It is also possible to estimate the number of strings that are weakly coupled up to a given string level $N_{m}=\sum_{k} k N_{(k, m)}$. For this it is sufficient to look at states with $k=1$, which have the strongest coupling for fixed $m$ (i.e. if the condition (5.5) is satisfied for states made with oscillators of frequency $k=1$, then it will be satisfied for all other states). Then $N_{m}=N_{(1, m)}=N_{0} \bar{N}_{(1, m)}$, so that eq. (5.5) becomes $m^{2} N_{0} \ll g^{2}$. Thus the number of strings $N_{s}$ whose excitations below a given level are weakly coupled is given by $N_{s}=O\left(g / \sqrt{N_{0}}\right)$. Similarly, for a given string $m$, all quantum string states with

$$
\alpha^{\prime} M^{2} \cong N_{m} \ll O\left(\frac{g^{2}}{m^{2}}\right)
$$


will be weakly coupled. States with $\alpha^{\prime} M^{2}>O\left(g^{2} / m^{2}\right)$ and $k=O(1)$ will not be subject to harmonic motion, since the motion will be mostly governed by $H_{1}$.

Let us now see what states survive in the limit $g^{2} \rightarrow 0$. By construction, $H_{1}$ is a sum of positive-definite terms; any state $|\Psi\rangle$ with $\left\langle\Psi\left|H_{1}\right| \Psi\right\rangle \neq 0$ will have infinite mass in the zero-coupling limit. In the limit $g^{2}=0=R_{0}^{2}$, with $T_{3} \rightarrow \infty$ so that $T=2 \pi R_{0} T_{3}$ remains fixed, the only states which survive are those containing excitations in a Cartan subspace of the area-preserving diffeomorphism algebra, so that $\left(\mathbf{n}_{1} \times \mathbf{n}_{2}\right)\left(\mathbf{n}_{3} \times \mathbf{n}_{4}\right)=0$ and $H_{1}$ gives no contribution. The $X_{(k, 0)}$ generates a Cartan subspace, implying that the type IIA superstring survives. In addition, just as in the toy model, there are excitations in other directions (having $\left\langle\Psi\left|H_{1}\right| \Psi\right\rangle=0$ ) which also remain. They include, in particular, the states constructed with the $X_{(0, m)}^{a}$ modes, the constant parts of the $X_{m}^{a}(\sigma)$ strings, which are responsible of the instability of the supermembrane.

\section{Membrane spectrum at infinite radius}

The eigenvalues of the mass operator are given by

$$
M^{2}=2 p_{0}^{+} p_{0}^{-}-\left(p_{0}^{a}\right)^{2}=2 H_{0}+2 H_{1}-\left(p_{0}^{a}\right)^{2}
$$

As is usual in Kaluza-Klein theories, $p_{0}^{10}$ has discrete eigenvalues $p_{0}^{10}=Q / R_{0}, Q \in \mathbf{Z}$, which from the ten-dimensional viewpoint are seen as Ramond-Ramond charges. The ten-dimensional mass operator is

$$
M_{10 D}^{2}=2 p_{0}^{+} p_{0}^{-}-\left(p_{0}^{i}\right)^{2}=M^{2}+\left(p_{0}^{10}\right)^{2}
$$

Let us now take the limit $R_{0} \rightarrow \infty, T_{3} \rightarrow 0$, with $T=2 \pi R_{0} T_{3}$ fixed. In this limit, $g^{2} \rightarrow \infty$, so the term $H_{1}$ can be dropped, and the fields can be expanded as in eq. (3.10). The (bosonic part of the) mass spectrum takes the form

$$
\frac{1}{2} \alpha^{\prime} M^{2}=\frac{1}{2} \alpha^{\prime} \sum_{m \neq 0} p_{m}^{a} p_{-m}^{a}+\sum_{m=-\infty}^{\infty} \sum_{k=1}^{\infty}\left[\alpha_{(-k,-m)}^{i} \alpha_{(k, m)}^{i}+\tilde{\alpha}_{(-k,-m)}^{i} \tilde{\alpha}_{(k, m)}^{i}\right]
$$

In this limit, the fact that the standard membrane spectrum is continuous is simply understood: the $p_{m}^{i}=\int d \sigma P_{m}^{i}(\sigma)$ (center-of-mass momenta of $m \neq 0$ "strings") take continuous values, since they are governed by the free particle Hamiltonian $H_{\text {free }}=\frac{1}{2} p_{m}^{i} p_{-m}^{i}=$ $\frac{1}{2}\left(p_{m}^{(1)}\right)^{2}+\frac{1}{2}\left(p_{m}^{(2)}\right)^{2}$, where $p_{ \pm m}=p_{m}^{(1)} \pm i p_{m}^{(1)}$. 
It is interesting to discuss the spectrum of a truncated Hamiltonian, defined by (3.6), (3.7) by setting the constant parts in $\sigma$ of the fields $X_{m}^{a}(\sigma), P_{m}^{a}(\sigma)$ to zero. Now no flat direction remains in the potential, and the motion of all modes is bounded. The mass spectrum is discrete for all radii and, in the infinite radius limit, takes the simple form

$$
\frac{1}{2} \alpha^{\prime} M^{2}=\mathbf{N}+\tilde{\mathbf{N}},
$$

where the bosonic part of $\mathbf{N}, \tilde{\mathbf{N}}$ can be read from eq. (6.2).

Let us now restore the fermion contributions in the formulas. Inserting the expansion

$$
\sqrt{p_{0}^{+}} \theta^{\alpha}(\sigma, \rho)=\sum_{m} S_{m}^{\alpha}(\sigma) e^{i m \rho}
$$

in $H_{\mathrm{F}}$, we get (see eq. 2.5))

$$
H_{\mathrm{F}}=T \int d \sigma\left[\sum_{m} \bar{S}_{-m} \Gamma^{10} \partial_{\sigma} S_{m}-\sum_{m, n} \frac{i}{R_{0}} \bar{S}_{-m-n} \Gamma^{a}\left(n \partial_{\sigma} X_{m}^{a} S_{n}-m X_{m}^{a} \partial_{\sigma} S_{n}\right)\right] .
$$

As $R_{0} \rightarrow \infty$, the second term disappears and the Hamitonian reduces to that of a free system. The ten-dimensional chiralities of the fermions are those of type IIA superstring theory. The complete mass formula is thus given by eq. (6.3), with $\mathbf{N}, \tilde{\mathbf{N}}$ containing the standard Green-Schwarz fermion-mode operators $S_{(k, m)}^{r}, \tilde{S}_{(k, m)}^{r}, r=1, \ldots, 8$, for the different strings parametrized by $m$, that is

$$
\begin{gathered}
\mathbf{N}=\sum_{m=-\infty}^{\infty} \sum_{k=1}^{\infty}\left[\alpha_{(-k,-m)}^{i} \alpha_{(k, m)}^{i}+k S_{(-k,-m)}^{r} S_{(k, m)}^{r}\right], \\
\left\{S_{(k, m)}^{r}, S_{\left(k^{\prime}, m^{\prime}\right)}^{s}\right\}=\delta_{m+m^{\prime}} \delta_{k+k^{\prime}} \delta^{r s},
\end{gathered}
$$

and a similar expression for $\tilde{\mathbf{N}}$. The zero-point energy cancels out as in the usual GreenSchwarz light-cone formalism of the superstring.

The physical Hilbert space is spanned by states made of the transverse excitations $\alpha_{(-k, m)}^{i}, \tilde{\alpha}_{(-k, m)}^{i}$ and $S_{(-k, m)}^{r}, \tilde{S}_{(-k, m)}^{i}$, with $k>0$ and $m \in \mathbf{Z}$. In addition, physical states must obey the level matching conditions:

$$
\mathbf{N}=\tilde{\mathbf{N}}, \quad \mathbf{N}^{+}-\mathbf{N}^{-}=Q
$$

The definition of the operators $\mathbf{N}^{+}, \mathbf{N}^{-}$is given in the appendix, where the derivation of conditions (6.7) is given. The first of these conditions plays the same role as the standard 
Virasoro level-matching condition of superstring theory. The second condition is new and it has no analogue in superstring theory. It is associated with translational invariance in the $\rho$ direction. Note that physical states with Ramond-Ramond charge $Q \neq 0$ necessarily involve excitations in the $\rho$ direction, i.e. they have $\mathbf{N}^{+} \neq 0$ or $\mathbf{N}^{-} \neq 0$.

It may seem surprising that in the infinite radius limit the membrane spectrum takes the simple form $(6.3)$, with the constraints $(6.7)$. However, it should be stressed that this is the spectrum for a membrane that wraps around the compact dimension, in the specific limit $T_{3} \rightarrow 0, R_{0} \rightarrow \infty$, with $R_{0} T_{3}$ fixed. In this limit the quartic terms in the potential are negligible in relation to the quadratic terms. For a membrane that does not wrap around $S^{1}$, there is no quadratic term and at any radius the dynamics is governed by the quartic terms. Note also that this membrane configuration does not give extra contributions to the energy. For a target space $\mathbf{R}^{9} \times S^{1} \times S^{1}$, it is possible to have the topologically nontrivial membrane winding number (2.12), which gives a contribution to the mass squared proportional to $T_{3}^{2} w_{0}^{2} R_{9}^{2} R_{10}^{2}$, as in eq. (2.13). It is of interest to study the spectrum in the case $\mathbf{R}^{9} \times S^{1} \times S^{1}$, since it will allow a comparison between the BPS excitation spectrum of type IIB superstring theory on $\mathbf{R}^{9} \times S^{1}$ and the M-theory counterpart, given in terms of wrapped 2-branes. 1 The correspondence between Kaluza-Klein modes and winding states of type IIB and M-theory was investigated by Schwarz (see [2] and additional references there).

\section{Consequences for M-theory}

What has been done in the previous sections may also be interpreted as follows. Suppose we start from type IIA superstring theory, imagining that this should arise as the zero-radius limit of some eleven-dimensional theory on $\mathbf{R}^{10} \times S^{1}$. We then turn on a small radius $R_{0}=\epsilon$, and inquire what kind of new degrees of freedom could be consistently added. The double-dimensional reduction concretely indicates that we should consider a 2-brane with one leg wrapped around the compact direction. However, we have seen that there are states made of mode operators associated with Cartan directions (for which $\left\langle\Psi\left|H_{1}\right| \Psi\right\rangle=0$, see eq. (5.3)), which can never emerge at $R_{0}=\epsilon$ if they were not

1 This calculation has been recently carried out in [12], and an exact matching of the corresponding BPS spectra was found. 
already present at $R_{0}=0$. In particular, wave packets constructed with the zero modes $X_{(0, m)}^{i}, P_{(0, m)}^{i}$. These zero modes are part of the fundamental scalar fields of the $(2+1)$ dimensional theory, and they cannot be excluded from the Hamiltonian, unless there is a kinematical or dynamical reason for this 2

It may be worth emphasizing that, unlike the spectrum of superstring theory, the spectrum (6.3) contains the infinite tower of Kaluza-Klein quantum states carrying RamondRamond charges. For small radius, the ten-dimensional mass operator 6.1) will be of the form

$$
\frac{1}{2} \alpha^{\prime} M_{10 \mathrm{D}}^{2}=\frac{Q^{2}}{2 g^{2}}+\mathbf{N}+\tilde{\mathbf{N}}+\alpha^{\prime} H_{1}, \quad g^{2}=R_{0}^{2} / \alpha^{\prime},
$$

where the $m \neq 0$ part of $\mathbf{N}, \tilde{\mathbf{N}}$ will be expressed in terms of $X_{(k, m)}^{i}, P_{(k, m)}^{a}$, as in eq. (5.2). Quantum states with $Q=0$ containing excitations of $a_{(k, m)}^{\dagger}$ with $m \neq 0$ will have a mass of order $1 / g$, originating from contributions of $H_{1}$. In addition, there are Kaluza-Klein states with $Q \neq 0$, which have $\sqrt{\alpha^{\prime}} M_{10 \mathrm{D}}=O(1 / g)$. Note that they must contain excitations with $m \neq 0$ in order to satisfy the second constraint in eq. (6.7). These states are presumably candidates for D0-branes and 1-branes of the type IIA superstring theory.

It would also be interesting to investigate the zero-mode problem in terms of dual actions. In particular, the duality between D2-brane action and the supermembrane action suggests that a more adequate description for the small radius limit could be in terms of a Born-Infeld type action, where the variables include world-volume vectors [14]. In this small $g^{2}$ limit, one could alternatively consider the 5-brane action. Another possibility is that the extra modes are just confined (with energy gaps $M$ of order $1 / g$ ) thanks to $\sigma$-model quantum corrections, which should be important for $R_{0}<\left(T_{3}\right)^{-1 / 3}$. Unfortunately, little is known about higher-derivative ( $\kappa$-invariant) extensions of the supermembrane action, but it would certainly be useful to have a tractable example.

2 Interestingly, in open string theory, there is a consistent way to quantize strings in which the center-of-mass remains fixed. This is done by imposing Dirichlet boundary conditions at the endpoints [13]. As a result, the center of mass, $x=\frac{1}{\pi} \int d \sigma X(\sigma, \tau)$ is $\tau$ independent. Some sort of closed string analogue (e.g. where the $p_{m}^{a}=\int d \sigma P_{m}^{a}(\sigma)$ are forced to be zero) may be of relevance to the present case. 


\section{Appendix A. Global constraints and the physical Hilbert space}

In the light-cone gauge, the coordinate $X^{+}$is set to $X^{+}=p_{0}^{+} \tau$, and $X^{-}$is determined from the constraints in terms of the other cordinates (see e.g. [9-11]):

$$
\partial_{\bar{a}} X^{-}=\frac{1}{p_{0}^{+}} \partial_{\bar{a}} X^{a} \dot{X}^{a}, \quad \bar{a}=\sigma, \rho .
$$

By taking the curl, one gets the condition

$$
\left\{X^{a}, \dot{X}^{a}\right\}=\partial_{\sigma} X^{a} \partial_{\rho} \dot{X}^{a}-\partial_{\rho} X^{a} \partial_{\sigma} \dot{X}^{a} \equiv 0
$$

or, in phase-space variables,

$$
\left\{X^{a}, P^{a}\right\} \equiv 0
$$

The Fourier components

$$
L_{\mathbf{n}}=\frac{1}{4 \pi^{2}} \int d \sigma d \rho Y^{\mathbf{n}}\left\{X^{a}, P^{a}\right\}
$$

are generators of the algebra of area-preserving diffeomorphisms (2.2). By a gauge transformation we can always rotate one of the coordinates, say $X^{10}$, into the Cartan subspace, as in eq. (3.1). Then eq. (A.3) takes the form

$$
L_{\mathbf{m}}^{\mathrm{T}}=-\sum_{\mathbf{n}^{\prime}} \sum_{\mathbf{n} \in K} X_{\mathbf{n}}^{10} P_{\mathbf{n}^{\prime}}^{10} f_{\mathbf{n n}^{\prime} \mathbf{m}}, \quad L_{\mathbf{m}}^{\mathrm{T}} \equiv \frac{1}{4 \pi^{2}} \int d \sigma d \rho Y^{\mathbf{m}}\left\{X^{i}, P^{i}\right\} .
$$

We note that the $P_{\mathbf{n}^{\prime}}^{10}, \mathbf{n}^{\prime} \in K$, are absent from this formula. The constraint A.5 determines $\tilde{P}^{10}(\sigma, \rho) \equiv \sum_{\mathbf{n} \notin K} P_{\mathbf{n}}^{10} Y_{\mathbf{n}}(\sigma, \rho)$ in terms of the $X^{i}, P^{i}$ and $X_{K}^{10}$. By formally inverting eq. (A.5), one gets

$$
\tilde{P}_{\mathbf{n}}^{10}=\left\{F^{-1}\right\}_{\mathbf{m n}} L_{\mathbf{m}}^{\mathrm{T}}, \quad F_{\mathbf{n m}}=-\sum_{\mathbf{n}^{\prime} \in K} X_{\mathbf{n}^{\prime}}^{10} f_{\mathbf{n}^{\prime} \mathbf{n m}} .
$$

The determinant of $F$ vanishes when some of the eigenvalues of $X_{\mathbf{n}^{\prime}}^{10}$ coincide, i.e. at the boundary of the Weyl chamber [6]. In the present case of a membrane wrapped on $\mathbf{R}^{10} \times S^{1}$,

$$
X^{10}=R_{0} \rho+\tilde{X}^{10}(\rho),
$$

and for small oscillation amplitudes, $\left\langle\left|\partial_{\rho} \tilde{X}^{10}\right|\right\rangle \ll R_{0}$, the relation (A.5) is always invertible, and a simple solution of the constraint eq. (A.2) can be found. Equation (A.2) becomes

$$
\left\{X^{i}, P^{i}\right\}=\left(R_{0}+\partial_{\rho} \tilde{X}^{10}\right) \partial_{\sigma} \tilde{P}^{10}
$$


In the $R_{0} \rightarrow \infty$ limit (with fixed winding around the compact direction), this reduces to

$$
\left\{X^{i}, P^{i}\right\} \cong R_{0} \partial_{\sigma} \tilde{P}^{10},
$$

which determines $\tilde{P}^{10}$ in terms of transverse coordinates. In the opposite limit $R_{0} \rightarrow 0$, there are singular boundary points which need to be treated with care. The discussion of instability modes is nevertheless not affected, since one can always choose suitable wave packets with support in the interior of the Weyl chamber [6].

Since $X^{-}$is single-valued, eq. (A.1) also implies the global constraints

$$
\begin{aligned}
& \mathbf{P}^{(\sigma)}=\frac{1}{2 \pi \alpha^{\prime}} \int_{0}^{2 \pi} d \sigma \partial_{\sigma} X^{a} \dot{X}^{a} \equiv 0, \\
& \mathbf{P}^{(\rho)}=\frac{1}{2 \pi \alpha^{\prime}} \int_{0}^{2 \pi} d \rho \partial_{\rho} X^{a} \dot{X}^{a} \equiv 0 .
\end{aligned}
$$

The operators $\mathbf{P}^{(\sigma)}, \mathbf{P}^{(\rho)}$ generate translations in $\sigma$ and $\rho$, respectively. By virtue of eq. (A.2), the integrals in (A.9), A.10) are independent of the contours. In particular, one readily checks that

$$
\partial_{\rho} \mathbf{P}^{(\sigma)}=0, \quad \partial_{\sigma} \mathbf{P}^{(\rho)}=0 .
$$

Let us now compute $\mathbf{P}^{(\sigma)}$ and $\mathbf{P}^{(\rho)}$ to leading order in the large-radius limit. By making use of the properties (A.11), we can write $\mathbf{P}^{(\sigma)}, \mathbf{P}^{(\rho)}$ in the more convenient form:

$$
\begin{aligned}
& \mathbf{P}^{(\sigma)}=\frac{1}{4 \pi^{2} \alpha^{\prime}} \int_{0}^{2 \pi} d \rho \int_{0}^{2 \pi} d \sigma \partial_{\sigma} X^{a} \dot{X}^{a}, \\
& \mathbf{P}^{(\rho)}=\frac{1}{4 \pi^{2} \alpha^{\prime}} \int_{0}^{2 \pi} d \sigma \int_{0}^{2 \pi} d \rho \partial_{\rho} X^{a} \dot{X}^{a} .
\end{aligned}
$$

Inserting eqs. (3.5), (3.10) in eqs. (A.12) and (A.13), after some straightforward algebra, we find the following expressions:

$$
\begin{gathered}
\mathbf{P}^{(\sigma)}=-\sum_{m=-\infty}^{\infty} \sum_{k=1}^{\infty}\left[\alpha_{(-k,-m)}^{i} \alpha_{(k, m)}^{i}-\tilde{\alpha}_{(-k,-m)}^{i} \tilde{\alpha}_{(k, m)}^{i}\right], \\
\mathbf{P}^{(\rho)}=Q+i \sum_{m=1}^{\infty} m\left(x_{m}^{i} p_{-m}^{i}-x_{-m}^{i} p_{m}^{i}\right)-\sum_{m, k=1}^{\infty} \frac{m}{k}\left[\alpha_{(-k,-m)}^{i} \alpha_{(k, m)}^{i}\right. \\
\left.+\tilde{\alpha}_{(-k,-m)}^{i} \tilde{\alpha}_{(k, m)}^{i}-\alpha_{(-k, m)}^{i} \alpha_{(k,-m)}^{i}-\tilde{\alpha}_{(-k, m)}^{i} \tilde{\alpha}_{(k,-m)}^{i}\right],
\end{gathered}
$$


where we have used

$$
X^{10}=R_{0} \rho+\sum_{m}\left(x_{m}^{10}+\alpha^{\prime} p_{m}^{10} \tau\right) e^{i m \rho}, \quad p_{0}^{10}=Q / R_{0} .
$$

It is convenient to introduce mode operators

$$
\beta_{(k, m)}^{i}=\frac{1}{\sqrt{|k|}} \alpha_{(k, m)}^{i}, \quad \tilde{\beta}_{(k, m)}^{i}=\frac{1}{\sqrt{|k|}} \tilde{\alpha}_{(k, m)}^{i},
$$

so that the commutators (3.11) and eqs. (A.14), (A.15) take a more symmetrical form under $k \leftrightarrow m$. Restoring the fermion contributions, the constraints (A.9), (A.10) become

$$
\begin{gathered}
\mathbf{N}=\tilde{\mathbf{N}}, \\
\mathbf{N}^{+}-\mathbf{N}^{-}=Q,
\end{gathered}
$$

where

$$
\begin{gathered}
\mathbf{N}=\sum_{m=-\infty}^{\infty} \sum_{k=1}^{\infty} k\left[\beta_{(-k,-m)}^{i} \beta_{(k, m)}^{i}+S_{(-k,-m)}^{r} S_{(k, m)}^{r}\right] \\
\tilde{\mathbf{N}}=\sum_{m=-\infty}^{\infty} \sum_{k=1}^{\infty} k\left[\tilde{\beta}_{(-k,-m)}^{i} \tilde{\beta}_{(k, m)}^{i}+\tilde{S}_{(-k,-m)}^{r} \tilde{S}_{(k, m)}^{r}\right] \\
\mathbf{N}^{+}=\mathbf{N}_{\mathbf{0}}+\sum_{m=1}^{\infty} \sum_{k=1}^{\infty} m\left[\beta_{(-k,-m)}^{i} \beta_{(k, m)}^{i}+S_{(-k,-m)}^{r} S_{(k, m)}^{r}\right. \\
\left.\quad+\tilde{\beta}_{(-k,-m)}^{i} \tilde{\beta}_{(k, m)}^{i}+\tilde{S}_{(-k,-m)}^{r} \tilde{S}_{(k, m)}^{r}\right] \\
\mathbf{N}^{-}=-\mathbf{N}_{\mathbf{0}}+\sum_{m=1}^{\infty} \sum_{k=1}^{\infty} m\left[\beta_{(-k, m)}^{i} \beta_{(k,-m)}^{i}+S_{(-k, m)}^{r} S_{(k,-m)}^{r}\right. \\
\left.\quad+\tilde{\beta}_{(-k, m)}^{i} \tilde{\beta}_{(k,-m)}^{i}+\tilde{S}_{(-k, m)}^{r} \tilde{S}_{(k,-m)}^{r}\right], \\
\mathbf{N}_{\mathbf{0}}=\frac{1}{2} \sum_{m=1}^{\infty} m\left[i\left(x_{-m}^{a} p_{m}^{a}-x_{m}^{a} p_{-m}^{a}\right)+\left[S_{(0, m)}^{r}, S_{(0,-m)}^{r}\right]+\left[\tilde{S}_{(0, m)}^{r}, \tilde{S}_{(0,-m)}^{r}\right]\right] .
\end{gathered}
$$

In the truncated theory, zero modes of $m \neq 0$ strings are dropped and one can set $\mathbf{N}_{\mathbf{0}}=0$.

The vacuum Fock state is defined by

$$
\begin{gathered}
\alpha_{(k, m)}^{i}|0\rangle=\tilde{\alpha}_{(k, m)}^{i}|0\rangle=0, \quad k>0, \\
S_{(k, m)}^{r}|0\rangle=\tilde{S}_{(k, m)}^{r}|0\rangle=0, \quad k>0, \\
p_{m}^{a}|0\rangle=0,
\end{gathered}
$$

for all $m$. The Fock space is generated by the states made by successive applications of the $\alpha_{(-k, m)}^{i}, S_{(-k, m)}^{r}, \tilde{\alpha}_{(-k, m)}^{i}, \tilde{S}_{(-k, m)}^{r}, k>0$ on the vacuum Fock state. Thus, in the $R_{0} \rightarrow \infty$ limit, we find that the Hilbert physical space is constituted of all states in the Fock space obeying the conditions (A.16) and (A.17). 


\section{References}

[1] E. Bergshoeff, E. Sezgin and P.K. Townsend, Phys. Lett. B189 (1987) 75; Ann. Phys. 185 (1988) 330.

[2] For a review, see J. Schwarz, hep-th/9607201.

[3] P.K. Townsend, Phys. Lett. B350 (1995) 184.

[4] E. Witten, Nucl. Phys. B443 (1995) 85 .

[5] M. Duff, P. Howe, T. Inami and K. Stelle, Phys. Lett. B191 (1987) 70.

[6] B. de Wit, M. Lüscher and H. Nicolai, Nucl. Phys. B320 (1989) 135.

[7] J.G. Russo, Phys. Lett. B (1997), hep-th/9609043.

[8] D. Fairlie, P. Fletcher and C. Zachos, Phys. Lett. B218 (1989) 203; J. Hoppe, Int. J. Mod. Phys. A4 (1989) 5235; B. de Wit, U. Marquard and H. Nicolai, Commun. Math. Phys. 128 (1990) 39.

[9] E. Bergshoeff, E. Sezgin and Y. Tanii, Nucl. Phys. B298 (1988) 187.

[10] B. de Wit, J. Hoppe and H. Nicolai, Nucl. Phys. B305 [FS 23] (1988) 545.

[11] M. Duff, T. Inami, C. Pope, E. Sezgin and K. Stelle, Nucl. Phys. B297 (1988) 515.

[12] J.G. Russo and A.A. Tseytlin, hep-th/9611047.

[13] J. Dai, R. Leigh and J. Polchinski, Mod. Phys. Lett. A4 (1989) 2073.

[14] P. Townsend, Phys. Lett. B373 (1996) 68; C. Schmidhuber, hep-th/9601003; A. Tseytlin, hep-th/9602064. 